\title{
1 Do Psychedelics Change Beliefs?
}

2

3 McGovern, H.T ${ }^{1}$, Leptourgos, P. ${ }^{2}$, Hutchinson, B.T. ${ }^{3}$, Corlett, P.R. ${ }^{2 *}$.

4

5 1. The University of Queensland, School of Psychology, Brisbane, QLD, Australia

6 2. Yale University, Department of Psychiatry, New Haven, CT, USA.

7 3. The Australian National University, Research School of Psychology, Canberra, ACT,

8 Australia

9

10 Abstract:

11 Renewed interest in psychedelics has reignited the debate about whether and how they

12 change human beliefs. In both the clinical and social-cognitive domains, psychedelic consumption may be accompanied by profound, and sometimes lasting, belief changes. We review these changes and their possible underlying mechanisms. Rather than inducing de novo beliefs, we argue psychedelics may instead change the impact of affect and of others' suggestions on how beliefs are imputed. Critically, we find that baseline beliefs (in the possible effects of psychedelics, for example) might color the acute effects of psychedelics as well as longer term changes. If we are to harness the apparent potential of psychedelics in the clinic and for human flourishing more generally, these possibilities must be addressed empirically.

Key Words: psychedelics, belief, affect

25

*Correspondence: Phil Corlett: philip.corlett@yale.edu. 


\section{The return of psychedelic science}

Since 2016, psychedelic science - particularly with human participants - has seen a significant revival. Studies administering psilocybin and lysergic-acid-diethlyemide (LSD) to healthy volunteers during functional magnetic resonance imaging promise insights into the mechanisms of consciousness. Psychedelic psychotherapy may help people with affective and addictive disorders ${ }^{1,2,3}$. Such benefits can occur for weeks, months, or even years following initial dosage $e^{1}$. These changes are posited to harness similar changes in beliefs (about self and others) that occur with cognitive behavioral therapy ${ }^{4}$. Outside of the clinical realm, psychedelics appear to change social and political beliefs ${ }^{5,6,7}$ and moral decision making ${ }^{8}$. However, disagreement remains about the locus of these effects in terms of perceptual and cognitive mechanisms ${ }^{8}$, or if belief change even occurs ${ }^{9}$.

Here we consider if and how psychedelics change human beliefs.

Humans have ingested psychedelic plants for much of our history ${ }^{10}$. They appear in Californian rock art from 500 years ago ${ }^{10}$. These plants are implicated in ritual and cultural practices, including shamanic ceremonies with the expressed purpose of modulating shamans' beliefs and those of their followers ${ }^{11}$. Albert Hoffman unwittingly exposed himself to LSD shortly after first synthesizing it in $1943^{11}$. He experienced the first LSD trip, describing it as both enlightening and frightening ${ }^{12}$. US-based scientists later explored LSD as a psychotherapeutic adjuvant and a model for psychosis - each of which implies an impact on beliefs and belief updating. With time, LSD became popular amongst the rising counterculture for its apparent consciousness expanding properties.

This is often assumed to be the main driver of its prohibition as a research tool. However, the truth is more nuanced ${ }^{13}$. At the time of LSD's greatest cultural impact, interest in its clinical potential within the company that manufactured the drug began to dwindle, largely because of the poorly controlled nature of the studies claiming breakthrough psychotherapeutic effects ${ }^{14,15}$. Around the same time, the Central Intelligence Agency became interested in the utility of psychedelics for mind-control and brainwashing. As part of their Cold-War efforts, the CIA funded research by Donald Hebb, Ewan Cameron, and 
60

61

62

63

64

65

66

67

68

69

70

71

72

73

74

75

76

77

78

79

80

81

82

83

84

85

86

87

88

89

90

91

John C. Lilly among others, providing grist for espionage thrillers, science-fiction fantasies and conspiracy theorizing for decades to come ${ }^{16}$.

Many years later, it remains unclear if psychedelics can change human beliefs. Given the resurgence of research, the moral and ethical implications of exposing human volunteers to compounds that may alter their beliefs and sense of self, warrant careful consideration. Are beliefs changing? And, given the risks, are we doing the best quality science in our attempts to find out? We believe the answers to these questions depend upon how we define beliefs.

\section{Do psychedelics change beliefs?}

The idea that psychedelics change beliefs originates from benefits observed in psychedelicassisted therapy for treatment of complex psychiatric disorders ${ }^{17}$. These benefits occur in patients for whom conventional antidepressants are ineffective ${ }^{18,19}$. They include decreased end-of-life depression and anxiety ${ }^{20,21}$, and reductions in substance abuse ${ }^{22,23,24}$. Such

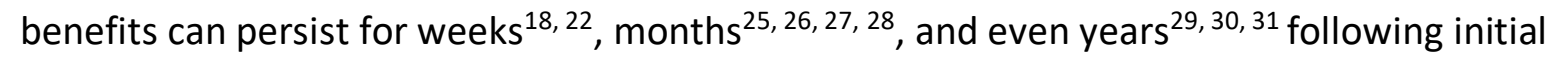
dosage (although extreme anxiety and fear can also occur, see ${ }^{30}$ ).

On top of the observed effects of psychedelic assisted therapy, people who have consumed psychedelics often report transformative experiences. They describe the experience as profoundly meaningful. Some groups find that a single dose of psilocybin produced the single most meaningful experience of the individual's life or among the top five ${ }^{24,32}$. In some cases, these "secondary" effects (i.e., over and above the clinical benefits) are so significant, that the primary clinical outcome effects are considered by the user as the least important consequence of administration ${ }^{40}$.

There also appear to be large effects in the non-clinical domain, including increases in altruism and prosociality ${ }^{31}$, connectedness ${ }^{33}$, enhanced concern for nature ${ }^{6,28}$, (also see $\left.{ }^{5}\right)$ and for other people ${ }^{38}$, changes in political orientation ${ }^{28}$, and decreases in pessimistic outlook $^{39}$. The findings highlight perhaps a fundamental appeal of psychedelic science - core psychological constructs usually considered stable across time seem to change in sometimes drastic ways ${ }^{37}$. It is perhaps unsurprising then that psychedelics' effects seem to include lasting changes to "beliefs" 28 , attitudes ${ }^{33}$, and personality $34,35,36$ (but see ${ }^{31}$ ). 
Here, we aim to unpack extant findings on the changes brought about by psychedelic consumption. Whilst the above effects of psychedelics appear hopeful, and we are desirous of them, we would like to urge caution. Such large changes may have multiple, often indirect, mechanisms. Among them, the fact that people expect these effects before consuming them, as well as possible collider bias. Here, we aim to define what beliefs are, and thence the locus of psychedelic effects.

\section{What is a belief?}

There is probably no agreed upon definition of belief. Despite that, discerning competing definitions of belief can provide boundary conditions for what is and is not changed following psychedelic consumption. We consider three alternatives: belief as propositional attitude, belief as association, and belief as inference and learning. Following this, we assess if beliefs, according to each definition, change with acute psychedelic administration, or in the aftermath of psychedelic exposure.

\section{Contemporary philosophers often characterize belief as a propositional attitude $41,42,43,44$.} Propositions are generally taken to be whatever it is a sentence expresses. Our attitude towards them captures whether we consider what is expressed in a sentence to be true. Propositional attitudes encapsulate religious and political beliefs. Yet they also describe more mundane beliefs about the state of the world that can be expressed linguistically. Propositional accounts can be shared with interlocutors, used to signal group membership, as well as form and benefit from coalitions ${ }^{45}$. Given this, propositional accounts of belief tend to align believing with having a capacity for language ${ }^{46,47,48}$. However, conceiving of propositions as causal Bayes nets has drawn some to speculate that propositional attitudes are not unique to humans (i.e., language is not required to form propositions ${ }^{49,50}$ ), although not without opposition ${ }^{51}$. Having said this, drawing a line between a proposition and simple association between two or more phenomena is not always entirely clear.

\section{(18)}

Associative accounts are often held to be in contradistinction to a propositional account ${ }^{54,55}$. An associative account of beliefs holds that believing is a function of the strength of association between mental features that represent causes, and similar features that represent effects ${ }^{56,57}$. These accounts have much in common with the mechanisms of 
associative learning theory, connectionist networks, and more recently deep learning, and find support in behavioral neuroscience ${ }^{58,59,60,61}$. For example, associations are thought to be strengthened as a function of prediction error minimization: events that violate predictions based on summed associative strength guide updates to that sum in order to minimize future error ${ }^{57,58,59}$. These error signals have been found in dopamine neurons during reward learning in primates ${ }^{60,61,62,63}$, during the learning of causal structure in rodents ${ }^{64,65,66}$, and during causal belief formation in humans with functional magnetic resonance imaging ${ }^{67,68}$ (see ${ }^{69}$ for review). Associative and propositional accounts may not actually differ. It remains possible that the structure of propositional attitudes is formed, and/or imputed from simpler associative learning.

A third account of beliefs is derived from an account of the brain as an inference engine. Here, practitioners conceive of neural and psychological processing in terms of beliefs - captured by a central (often Bayesian) calculus that trades off prior beliefs (expectations) with likelihoods (data), to arrive at posterior beliefs (conclusions) $)^{70,71}$. Crucially, the structure that carries belief here is a distribution of possible values some variable could take, with a mean and standard deviation, or precision - which implies a degree of confidence - in the values the belief might take. One means of implementing the computations that sweep from prior to posterior, via likelihood, involves the expectationmaximization algorithm, which allows distinction between inference and learning. Inference is the best explanation of sensory data based on the sum of prior beliefs, and learning is how those prior beliefs are updated and how they color future inference. The distinction between inference and learning is important for meaningful evaluation of psychedelic effects, as they are likely moderating mechanisms behind any changes to broader propositional beliefs ${ }^{70,71,72,73,74,75,76}$. In prior work we argue that psychedelics and other psychotomimetic interventions (like ketamine infusion or sensory deprivation) might acutely alter inference, without much impinging upon longer-term learning (which lasts beyond the metabolism and excretion of the drug $)^{74,75,76}$. Some argue that psychedelics induce hallucinations by increasing the weighting of priors ${ }^{74,75}$.

In sum, there are multiple ways of defining beliefs - including propositions, associations, inference, and learning (see Box 1 for further elucidation). Whether 
156

157

158

159

160

161

162

163

164

165

166

167

168

169

170

171

172

173

174

175

176

177

178

179

180

psychedelics change beliefs critically hinges on which definition of belief is ultimately evoked. It may appear that single doses of psychedelics alter propositional attitudes, but it is more likely that they alter associative learning and the weighting of prior beliefs.

Furthermore, we argue that baseline beliefs are critically important, both in terms of who volunteers for psychedelics studies (there may be a collider bias) and what expectations they bring to their study experiences. Psychedelic hallucinations could arise from a loosening of the constraining effects of prior beliefs on perceptual inference ${ }^{76}$. It may be that psychedelics broaden the possibility space - inducing entirely new prior beliefs perhaps expanding the possible beliefs an individual entertains. Below, we examine the extent to which beliefs are changed by psychedelic usage, by considering the evidence of belief change under each definition.

Figure 1. Spatial and temporal priors. (a.) Schematic illustration of contextual effects (spatial priors) in reading 77 . We can easily read the phrases: "Jack and Jill went up the hill" and "The last event was cancelled", although the words "went" and "event" look identical. The context helps identifying the most likely word, i.e., the best explanation of the sensory input. (b.) A graphical representation of the generative model for reading the first phrase in (a.). The spatial prior induced by the semantic context ( $Z$ ) disambiguates the inherently ambiguous shape $(X)$, giving rise to the most probable interpretation $(Y)$. (c.) In the brain, contextual effects (spatial priors) can be implemented as feedback signals from high-level to low-level areas. (d.) A continuous-report test for orientations, in which sequential effects (temporal priors) are often reported. (e.) When participants are presented with sequences of gratings, judgements are often biased towards the orientation presented (or perceived) on the previous trial. (f.) Neural implementation of temporal priors: a crucial role is played by the dynamics of local E-I networks. 
a.

fack and fill event up the hill

The last event was cancelled b.

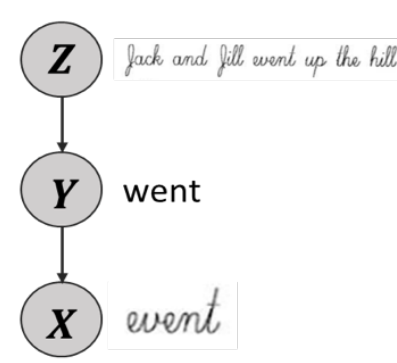

C.

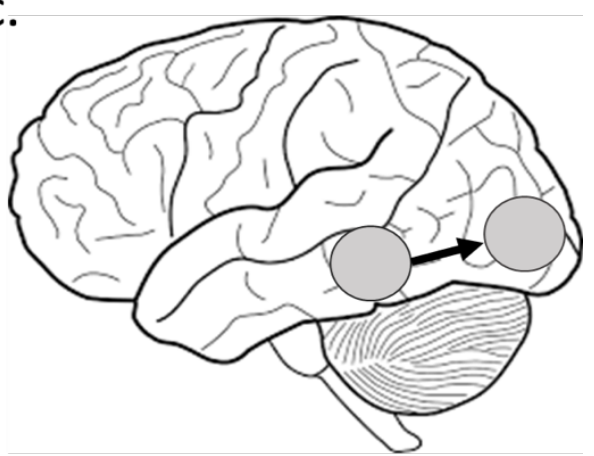

f.

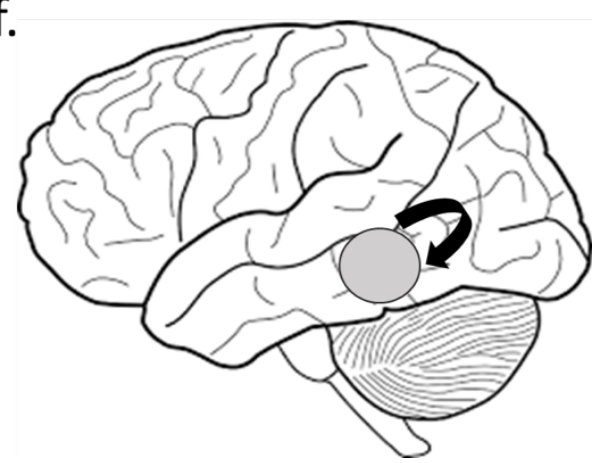

e.
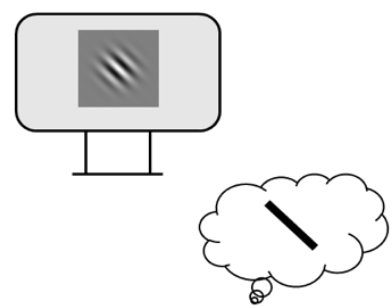
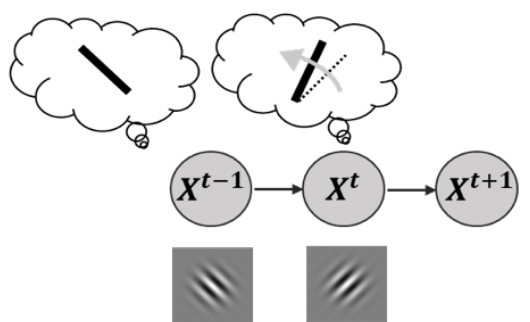

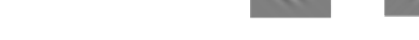

Box 1. Do psychedelics enhance or suppress priors?

Over the past two decades, the description of the brain as an "inference machine" gained significant support $78,79,80,81,82,83$. This framework posits that belief formation and (perceptual) decision making rest upon internal, hierarchical representations of the world, built by the brain over time. These generative models capture the world's causal structure, allowing the brain to generate prior expectations (priors) regarding future exteroceptive and interoceptive signals. New inputs are combined with the priors and, in turn, update the internal representations through inferences about the world's (or brain's or body's) new state. Crucially, all information is weighted according to its reliability, hence inferences are mainly driven by the most reliable cues. This weighted cue combination, together with the engagement of priors, ensure optimal actions and decisions, even when the sensory evidence is inherently ambiguous. However, an overreliance on priors can result in a detachment from one's sensations, bringing about aberrant percepts (hallucinations and illusions) and unshaken beliefs ${ }^{84,85,86}$. Could the effects of psychedelics be underscored by such strong priors? Or is the opposite - relaxed priors and overreliance on inputs - true?

To answer, one must first define "priors". In its most general sense, the term "prior" encompasses any available information about a certain aspect of the world that precedes (is not conditional on) sensory inputs. The very structure of our 4-dimensional world ( 3 spatial and 1 temporal dimensions) implies that all embodied brains must be 
equipped with two general types of priors: spatial (or hierarchical) and temporal priors ${ }^{87}$, 88,89 Spatial priors rely on causal links between variables of different abstraction and complexity, i.e., variables at different hierarchical levels (Figure 1a, b) 77,90 . Those priors include both pure spatial relationships (e.g., contextual effects or statistical properties of natural images; see Figure 1a) and more abstract relationships between variables (e.g., "if there is a cloud, it might rain"). They constitute top-down effects, mediated by long-range feedback connections ${ }^{91,92}$ and speak to the notion of cognitive penetrability, i.e., the idea that cognition can, in certain cases, affect perception ${ }^{93,94,95}$ (Figure 1c).

Temporal priors, unlike spatial priors, do not rely on cross-scale relationships between variables, but rather on the temporal statistics of each variable, laying the foundations for evidence accumulation ${ }^{77,96,97}$. The simplest instance of such a temporal prior is exemplified by a Markov chain (Figure 1e): the hierarchy is reduced to a single variable; whose value depends only on the very recent history (Markov property). Such dynamical models can account for simple sequential effects, including priming (or

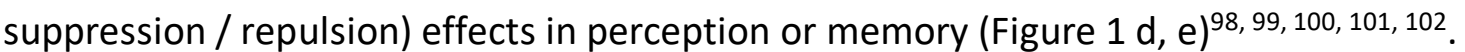
Importantly, although spatial and temporal priors are not totally distinguishable, the latter most likely rely less on long-range feedback connections and more on alterations in local microcircuits (e.g., neural gain changes or tuning shifts $92,102,103$; Figure 1f). Consequently, a strong (or weak) prior theory of psychedelics does not necessarily imply that spatial and temporal priors are equally affected. Given the present paper's focus on high-level beliefs, we will restrict ourselves to spatial / hierarchical priors.

Both the acute and the longer-term effects of psychedelics appear compatible with the strong- prior theory. Hallucinations, one of the most striking features of the acute psychedelic experience, have been related to an extreme reliance on prior expectations $^{84,86}$. In addition to that, the profound effects of set and setting ${ }^{104}$, including the cultural impact on the experience ${ }^{105}$, are compatible with enhanced top-down processing and amplified cognitive penetration. Furthermore, strong-prior theory is also in line with the finding that psychedelics do not alter strong pre-existing beliefs and, if anything, further strengthen them. Finally, in-lab experiments also support this theory.

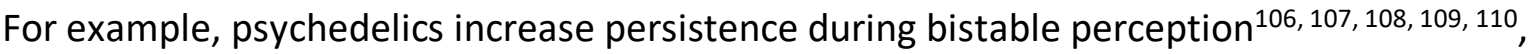


an effect that can be attributed to overcounted priors ${ }^{110}\left(\right.$ see $^{82,83}$ for overview of this model of cognition).

183

184

185

186

187

188

189

190

191

192

193

194

195

196

197

198

199

200

201

202

203

204

205

206

207

208

209

210

211

212

\section{Propositional Beliefs and Psychedelics}

Propositional beliefs are generally taken to be an attitude toward what a proposition expresses. They often take the form of religious, political, and philosophical beliefs. Several studies suggest changes in each of these domains following initial dosage. Generally, psychedelic usage is linked to more a more positive outlook $20,21,25,2632$. Other studies find long-term decreases in political authoritarianism ${ }^{28}$, more liberal attitudes ${ }^{5}$ and self-reported increases on pro-environmental stances ${ }^{6}$. This has led some to speculate that psychedelics carry potential for inducing long-term belief changes ${ }^{4}$, although this is not without dispute ${ }^{9}$ Propositional beliefs ostensibly changed by psychedelics are those expressed in the social domain - hence appearing as if they represent fundamental changes to belief structures.

\section{Social/Environmental Factors}

Propositional beliefs are largely tied to language. Given its importance in social cognition, language is critical for social interactions, so propositional accounts center the social functions of belief. We learn about others' beliefs, and we use that information to weight them as sources for our own belief evaluation on similar topics, and unrelated ones $^{111}$. Belief statements serve to identify individuals as potential friends or foes. Belief formation in the social arena is thus meta-cognitive, in that it entails representing one's own beliefs, our beliefs about those beliefs, and our beliefs about other's beliefs ${ }^{111}$. Given the social function of propositional beliefs, it is worth probing the impact of social setting in which propositional beliefs may be altered in non-psychedelic interventions. If people are shown to change propositional beliefs based on experimenter suggestion (in studies without psychedelics) then parsing whether reported changes occur due exclusively to psychedelic consumptions is paramount.

In non-psychedelic studies, humans show remarkable speed in updating their beliefs. Having been conditioned that a red and a blue square in compound predict an electric shock, that compound stimulus will elicit a galvanic skin response indicative of anticipatory fear. If an experimenter verbally states, "Blue is safe" and the squares are presented in 
213 isolation, red will continue to evoke increased skin conductance, whereas blue will not ${ }^{113}$.

214 Whilst the precise mechanisms underlying this kind-of learning (associative, propositional, 215 hybrid) and its relationship to conscious report, and the meta-cognitive state of claiming a belief, remain to be determined ${ }^{113}$, our point here is that effects of instructed learning parallel those of, and hence provide clues to, the effects of experimenter suggestion and social norms on shaping many of the reported outcomes (such as self-reported changes in beliefs) in psychedelic research.

A recent example provides a useful example. Here, researchers were interested in examining belief-updating under social pressure whilst participants were experiencing LSD or placebo ${ }^{114}$. Participants judged the aesthetic quality of art pictures. They were then confronted with a group norm (how others in the group typically judged that piece) and were subsequently invited to reevaluate their decision. Technically, these are attitudes, rather than beliefs - since they incorporate a valence judgment about the art, but they are belief-like in that they carry propositional features and are ostensibly based on what the person judges to be true. Based on the evidence discussed thus far, we might expect that conformity to the norm would increase under LSD. This was partially the case. However, not globally. On LSD, participants moved their judgements nearer to the norm, but only for attitudes that were less extreme, and already in range of the norm ${ }^{114}$. More extreme beliefs did not change under LSD or placebo. These findings show that acute LSD exposure strengthens relatively weak beliefs which are already present. More broadly, this suggests that it is social learning strategies which are subject to change under psychedelics. Assessment of how reliable others are may be perturbed such that we find their beliefs more salient in sculpting our own, more tentative, conclusions. In sum, it could be that we defer to others more in shaping our beliefs after psychedelics, rather than psychedelics changing our beliefs wholesale.

\section{Person Factors}

This leads to the next cluster of potential factors explaining observed belief change. Loading on the question of whether psychedelics change propositional beliefs is also whether the beliefs are strongly held. Weaker beliefs appear subject to change following consumption, but strong beliefs that precede consumption appear more resistant ${ }^{114}$. 
Second, when we draw conclusions about the mystical, spiritual, or political revelatory effects of psychedelics we should be mindful of baseline beliefs. The types of people who are drawn to volunteer for clinical research into psychedelic effects might be open to experiences and prone to certain beliefs (like alternative medicine and new age ideas) at baseline. Given what we know about task-elicited beliefs, these endogenous ideas and expectations will be the ones that manifest in the acute experience and perhaps strengthen even in the post-drug period. Indeed, recent research suggests LSD enhances suggestibility in healthy volunteers ${ }^{115}$, without enhancing creative imagination. Its effects on suggestion were greatest in people with higher trait conscientiousness ${ }^{115}$. That is, the themes and expectations that are primed before and during a psychedelic experience often manifest in the recipient's experiences. This has also been observed with other psychotomimetic drugs like ketamine ${ }^{116}$.

256

The drug itself may not even be necessary, as the placebo response is so strong with regards to the expected effects of psychedelics that no drug may be needed to induce them ${ }^{117}$. This is also true of anthropological studies. Ritual use of psychedelics often primes the experience, colors its aftermath, and infuses post-hoc interpretations, such that beliefs are changed - before and after the experience - though not necessarily through the pharmacological effects of the drug ${ }^{118}$. These studies further emphasize the importance of what an individual already believes in sculpting subsequent psychedelic experience and underscore the relevance of baseline beliefs when inferring psychedelics' effects on beliefs.

\section{Box 2. Is the subjective experience causally involved?}

How central are the acute subjective experiences associated with ingestion of psychedelics in bringing about positive changes? Several studies of psychedelics as therapeutics report correlations between the profundity of the psychedelic experience and outcome measures up to one-year post-exposure $20,22,24,29,31,35,119,120$.

Using a combination of clinical interview and survey response instruments such as the mysticism scale ${ }^{121}$, the states of consciousness questionnaire ${ }^{122}$, and the hallucinogen rating scale ${ }^{123}$ participants indicate the degree to which their experience was meaningful, spiritual, or elicited change in their lives. One such example is the 
persisting effects questionnaire ${ }^{124}$. The PEQ employs, in some versions, over 140 items exploring changes in attitudes about life and the self, mood, behavior, relationships, and spirituality, that the user attributes to the subjective experience itself. Participants consistently ascribe to the experience positive-rather than negative-changes in attitudes about life, mood, and behavior ${ }^{122}$, that are not only sustained, but increase over time 29,124 . The "mysticality" of the experience, broadly characterized by intense feelings of awe, ineffability, transcendence, and profundity ${ }^{124}$, is often measured with the psychometrically validated MEQ30 ${ }^{123}$. Scores on the MEQ30 correlate with reduced anxiety ${ }^{123}$ and depression scores ${ }^{123}$ in the weeks following the drug experience. Similar findings have been reported for alcohol ${ }^{118}$ and smoking dependence ${ }^{117}$. More profound psychedelic experiences predict reductions in self-reported drinking at one-month follow-up, reduced cravings, and increased self-efficacy in alcohol abstinence ${ }^{22}$. The personal meaning, spiritual significance, and changes in life satisfaction attributed to the experience also predict smoking outcomes including urinary cotinine levels (a metabolite of nicotine) at 30-month follow-up ${ }^{24}$.

Changes in personality induced by psychedelics are more varied. Earlier studies found no changes ${ }^{122}$, but when combined with additional data, changes in openness (as measured by the NEO-PI-R) were predicted by the acute intensity of the psychedelic experience ${ }^{35}$. Notably, these personality changes were sustained at one-year follow-up in those categorized as having a "complete" mystical experience (scoring $60 \%$ or higher on each subscale of the SOCQ) ${ }^{35}$. More recent studies ${ }^{119}$ find changes in neuroticism and extraversion at 3month follow-up that were predicted by measures of insightfulness, spirituality, and blissfulness and unity during the experience; whilst other studies find greater reductions in neuroticism at 6-months following ayahuasca ingestion, in those who scored higher on the MEQ30 ${ }^{36}$. Notably, there was no correlation between baseline neuroticism (i.e., measured prior to ingestion) and MEQ scores, suggesting the effect was driven through those experiences captured by the MEQ. Changes in well-being and life satisfaction (as measured via the PEQ) are also predicted at 6-month follow up by sores on the MEQ3020, 26, as are PEQ-measured positive changes in attitude, behavior, and life satisfaction with the intensity of the psychedelic experience at 12months following $\mathrm{LSD}^{31}$.

One possibility for delineating the causal role of mystical and connectedness experiences for the clinical response, would be to administer the psychedelic whilst participants were sedated, unconscious of the mystical experiences, though of course there are safety concerns. Furthermore, the choice of sedative agents will be key - many share neurochemical mechanisms with rapid-acting psychedelic antidepressants, and they may have similar antidepressant effects ${ }^{125,126}$. 
Ultimately, despite some intriguing correlations between features of the psychedelic experience and clinical outcomes, given the data on suggestion, conformity, and placebo responses (see text), we suggest causal conclusions regarding the role of mystical and connectedness experiences in long-term change may be premature.

265

266

267

268

269

270

271

272

273

274

275

276

277

278

279

280

281

282

283

284

285

286

287

288

289

290

291

\section{How could Psychedelics change beliefs? Association, Inference and Learning}

Whilst we see that the effects of psychedelics on propositional beliefs are tentative, they may have more profound effects on association, inference and learning. Acute intoxication with psychedelics can induce simple and complex visual hallucinations, potentially due to their excitation of $5-\mathrm{HT}_{2 \mathrm{~A}}$ receptors on pyramidal neurons and consequent glutamate release, resulting in altered prefrontal cortex activity ${ }^{1,127,128}$. The longer-term effects of hallucinations, and mystical psychedelic experiences on beliefs remain unclear. These possible lasting effects - beyond acute intoxication - have long been the subject of a moral panic. Do psychedelics induce a persistent psychotic state, characterized by uninvited and frightening flashbacks? The answer is certainly not uniformly. Although in some individuals (typically not those who volunteer for controlled clinical studies, who are pre-screened for safety, but rather those using recreationally) a persistent hallucinatory persistent perceptual disorder (HPPD) does occur ${ }^{129,130}$. It comprises simple, often geometric, visual hallucinations (Type I), sometimes with accompanying distress (Type II) ${ }^{129,130 .}$

Persistent visual hallucinations suggest that new perceptual prior beliefs are created by the psychedelic experience and sustained outside of the acute drug context; but since they do not typically invite narrative conclusions about alternate realities or alien agents, HPPD experiences do not appear to alter propositional beliefs. Indeed, much like the hallucinations that occur in neurological illnesses, most people with HPPD retain insight into the unreality of their hallucinations ${ }^{130}$. They do not invite new metaphysical propositional beliefs, but they might instead reflect associative learning of new (or differently weighted) prior beliefs ${ }^{131}$.

Intriguingly, in the work previously outlined on belief-updating under social pressure and LSD administration, ketanserin blocked the effects of LSD on conformity bias for beliefs 
that were near to (but not far from) the group norm ${ }^{114}$. This centers the $5 \mathrm{HT}_{2 a}$ receptor in the norm-conforming impact of LSD (though ketanserin has activity at other monoamine receptors, notably dopamine and noradrenaline $33,34,132,133,134,135$, ). In recent preclinical work, the $5 \mathrm{HT} 2 \mathrm{a}$ receptor in rodent medial prefrontal cortex is identified as a locus of memory reactivation and reconsolidation $40,136,137$. In previous work we argue that memory reconsolidation may underwrite the retention and strengthening of beliefs in the face of contradictory evidence in service of delusion maintenance ${ }^{41}$. Thus, the prior beliefs that receive spurious support under psychedelics may do so through mPFC 5HT2a receptor stimulation and reconsolidation mediated strengthening ${ }^{136,137}$. The longer-term consolidation and retention of this strengthening is deserving of further clinical and preclinical investigation.

Future studies could also assess the impact of psychedelic on social learning strategies more directly: Do psychedelics first impact non-social processes and thence social, or vice-versa? Do they change the relative weighting of social over non-social information? Do they increase the salience of others' beliefs? Do these changes occur as a function of changes in the reliability of social relative non-social information ${ }^{138}$ ? Likewise, is the dissolution of the usual boundary between us and others, a cause, a consequence, or a correlate of the impact of psychedelics on social learning strategies? Resolving the tension between social and nonsocial processes will have implications for the clinical impact of psychedelics. For example, individual differences in sensitivity to social and non-social learning strategies and their combination may explain why psychedelics can induce paranoia in some participants, ${ }^{139}$ and resolve anxieties in others. It could be that insofar as psychedelics alter learning strategies, they appear to alter propositional beliefs.

\section{Summary, Challenges, and Future Directions}

So far, we've suggested that if psychedelics change "beliefs", they do so largely through experimenter suggestion and the pre-existing psychology of the individual, and further, that the definition of belief one adopts (associative, propositional, inferential) is crucial to addressing the question. We suggest evidence is equivocal as to whether 
psychedelics change propositional beliefs directly. However, associations, inference, and learning may fair better.

Given that acute psychedelic experiences seem to alleviate depression, anxiety, and aid smoking cessation, in a manner that correlates with the intensity of acute mystical experiences at the time of drug exposure (see Box 2), it might be argued that these changes

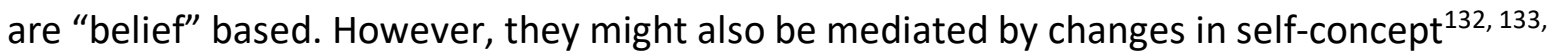
135. A salient feature of the psychedelic experience is a loss of sense of self, a dissolution of ego boundaries, and an experience of enhanced connectedness with other people, animals, living organisms, and celestial bodies. The capacity for psychedelics to engender these feelings may be at the core of their therapeutic impact.

The combined effects of self-dissolution and enhanced feelings of connectedness may underlie increases in positive mood and observed increases in openness. Having said this, it is worth remembering that volunteer themselves for psychedelic research may be higher in such traits in the first instance. We must also consider the powerful placebo effects that characterize psychedelic experiences and anti-depressant and anti-anxiety interventions. Appropriate placebo control thus remains a challenge in psychedelic science. These preliminary observations with anesthetics seem to suggest that mystical experiences are not necessary for clinical effects, though we should pay close attention to the experiences people report upon emergence from the anesthesia.

If connectedness experiences do increase under acute psychedelics, and that those experiences sustain a change in beliefs about the connectedness of people and animals, we might expect psychedelics to change participants' beliefs about pan-psychism: that living and non-living things possess an essence, a varying degree of consciousness, even the inanimate ${ }^{140}$. We might expect that people who reach such conclusions following psychedelics would become more inclined to recycle (treating the interconnected world with more respect) or more interested in consuming a vegan or vegetarian diet.

Environmental beliefs have, since the 1990s been more associated with the political left - at least in the US. It may be that people become more left leaning because of their psychedelic experiences. However, the relationships between environmental and new-age interests and political beliefs are not so straightforward. Right-wing groups have historically been 
interested in new-age ideas, including yoga, and the Aum Shinrikyo Buddhist cult which began by emphasizing ascetic Yoga practices, with time began to administer psychedelics to its followers, and, as a group began to espouse increasingly right-wing ideas ${ }^{141}$.

We are not suggesting that psychedelic use leads to cult membership, nor are we asserting that psychedelics induce extreme left- or right-wing belief. Rather, we are questioning whether, based on the empirical and anecdotal data, the suggestions that psychedelics necessarily lead to enlightenment, good outcomes, or more left-wing, collectivist ideals, ought to be taken seriously. We suggest not. Future work might explore the dimension of spiritual narcissism in psychedelic science. Briefly, those drawn to new age practices like yoga, may evince an enhanced self-focus, rather than ego-dissolution. This may manifest in beliefs and actions that are contrary to the tenets of the practice in which they are engaging. People who seek enlightenment through psychedelics might be likewise prone. This should be examined empirically.

\section{CONCLUSION}

We have explored whether and how psychedelics facilitate lasting belief change. Whilst we are intrigued and appreciate the potential that brief interventions with psychedelics might have in the psychiatric clinic and for human flourishing more broadly, like Sandoz in the 1960s we are circumspect. We call for better controlled trials that assess baseline beliefs and expectations prior to randomizing individuals to psychedelic interventions, we suggest a broad range of psychoactive control interventions, parametric manipulations of psychedelic settings, and, perhaps the most extreme, interventions in the absence of consciousness, to assay the causal role of mystical experiences in the apparent long-term effects of psychedelics. These compounds retain their fascination for humans precisely because they proffer insights into the meta-physical. To harness those insights most effectively and administer them ethically, we believe much more careful experimentation is necessary.

More fundamentally, we think a systematic assessment of beliefs and belief updating may be enlightening. We have formal mathematical models of beliefs that span computational, algorithmic, and implementational levels of analysis, which can be readily applied to neural and behavioral data gathered before, during, and after psychedelic 
387

388

389

390

391

392

393

394

395

396

397

398

399

400

401

402

403

404

405

406

407

408

409

410

411

412

413

414

415

416

417

418

419

infusions. Working within such frameworks will address many of the issues we raise presently. There are rudimentary questions yet to be answered in the neuroscience of belief, central of which, whether brains deal in probabilistic distributions, binary variables, or both. Addressing these questions will yield further means to assert whether associative, propositional, and/or inferential mechanisms are involved. We are hopeful that, much like how cognitive neuropsychiatry illuminates both lesion cases, and healthy function, psychedelic science may likewise inform the mechanisms of belief formation and updating.

\section{REFERENCES}

${ }^{1}$ Vollenweider, F. X., \& Preller, K. H. (2020). Psychedelic drugs: neurobiology and potential for treatment of psychiatric disorders. Nature Reviews Neuroscience, 21(11), 611-624.

${ }^{2}$ Muttoni, S., Ardissino, M., \& John, C. (2019). Classical psychedelics for the treatment of depression and anxiety: a systematic review. Journal of affective disorders, 258, 11-24.

${ }^{3}$ Patra, S. (2016). Return of the psychedelics: Psilocybin for treatment resistant depression. Asian Journal of Psychiatry, 24, 51-52.

${ }^{4}$ Carhart-Harris, R. L., \& Friston, K. J. (2019). REBUS and the anarchic brain: toward a unified model of the brain action of psychedelics. Pharmacological reviews, 71(3), 316-344.

${ }^{5}$ Nour, M. M., Evans, L., \& Carhart-Harris, R. L. (2017). Psychedelics, personality and political perspectives. Journal of psychoactive drugs, 49(3), 182-191.

${ }^{6}$ Forstmann, M., \& Sagioglou, C. (2017). Lifetime experience with (classic) psychedelics predicts pro-environmental behavior through an increase in nature relatedness. Journal of Psychopharmacology, 31(8), 975-988.

${ }^{7}$ Rifkin, B. D., Maraver, M. J., \& Colzato, L. S. (2020). Microdosing psychedelics as cognitive and emotional enhancers. Psychology of Consciousness: Theory, Research, and Practice, 7(3), 316-329. 
420

421

422

423

424

425

426

427

428

429

430

431

432

433

434

435

436

437

438

439

440

441

442

443

444

445

446

447

448

449

450

451

452

453

454

${ }^{8}$ Pokorny, T., Duerler, P., Seifritz, E., Vollenweider, F. X., \& Preller, K. H. (2020). LSD acutely impairs working memory, executive functions, and cognitive flexibility, but not risk-based decision-making. Psychological medicine, 50(13), 2255-2264.

${ }^{9}$ Johnson, M.W., \& Yaden, D.B. (2020). There's No Good Evidence That Psychedelics Can Change Your Politics or Religion. Scientific American.

${ }^{10}$ Robinson, D. W., Brown, K., McMenemy, M., Dennany, L., Baker, M. J., Allan, P., Cartwtight, C., Bernard, J., Sturt, F., Kotoula, E., Jazwa, C., Gill, K.M., Randolph-Quinney, P., Ash, T., Bedford, C., Gandy, D., Armstrong, M., Miles, J., \& Haviland, D. (2020). Datura quids at Pinwheel Cave, California, provide unambiguous confirmation of the ingestion of hallucinogens at a rock art site. Proceedings of the National Academy of Sciences, 117(49), 31026-31037.

${ }^{11}$ Powers, A. R., \& Corlett, P. R. (2018). Shamanism and psychosis: Shared mechanisms?. The Behavioral and Brain Sciences, 41, e83.

${ }^{12}$ Aday, J. S., Bloesch, E. K., \& Davoli, C. C. (2019). Beyond LSD: A broader psychedelic zeitgeist during the early to mid-20th century. Journal of psychoactive drugs, 51(3), 210-217.

${ }^{13}$ Sessa, B. (2012). The psychedelic renaissance: Reassessing the role of psychedelic drugs in 21st century psychiatry and society. Muswell Hill Press.

${ }^{14}$ Oram, M. (2014). Efficacy and enlightenment: LSD psychotherapy and the drug amendments of 1962. Journal of the History of Medicine and Allied Sciences, 69(2), 221-250.

${ }^{15}$ Dyck E. (2015). LSD: a new treatment emerging from the past. CMAJ : Canadian Medical Association journal = journal de l'Association medicale canadienne, 187(14), 1079-1080. Advance online publication. https://doi.org/10.1503/cmaj.141358.

${ }^{16}$ Ulrich, R. F., \& Patten, B. M. (1991). The rise, decline, and fall of LSD. Perspectives in biology and medicine, 34(4), 561-578. 
455

456

457

458

459

460

461

462

463

464

465

466

467

468

469

470

471

472

473

474

475

476

477

478

479

480

481

482

483

484

485
${ }^{17}$ Williams, M., \& Warner, M. (2019). The psychedelic renaissance. Australasian Science, 40(2), 16-18.

${ }^{18}$ Carhart-Harris, R. L., Bolstridge, M., Rucker, J., Day, C. M., Erritzoe, D., Kaelen, M., ... \& Nutt, D. J. (2016). Psilocybin with psychological support for treatment-resistant depression: an open-label feasibility study. The Lancet Psychiatry, 3(7), 619-627.

${ }^{19}$ Carhart-Harris, R. L., Bolstridge, M., Day, C. M. J., Rucker, J., Watts, R., Erritzoe, D. E., ... \& Nutt, D. J. (2018). Psilocybin with psychological support for treatment-resistant depression: six-month follow-up. Psychopharmacology, 235(2), 399-408.

${ }^{20}$ Griffiths, R. R., Johnson, M. W., Carducci, M. A., Umbricht, A., Richards, W. A., Richards, B. D., Jesse, R., Maclean, K.A., Barrett, F.S., Cosimano, M.P., \& Klinedinst, M. A. (2016).

Psilocybin produces substantial and sustained decreases in depression and anxiety in patients with life-threatening cancer: A randomized double-blind trial. Journal of psychopharmacology, 30(12), 1181-1197.

${ }^{21}$ Ross, S., Bossis, A., Guss, J., Agin-Liebes, G., Malone, T., Cohen, B., Mennenga, S. E., Belser, A., Kalliontzi, K., Babb, J., Su, Z., Corby, P., \& Schmidt, B. L. (2016). Rapid and sustained symptom reduction following psilocybin treatment for anxiety and depression in patients with life-threatening cancer: a randomized controlled trial. Journal of psychopharmacology (Oxford, England), 30(12), 1165-1180.

${ }^{22}$ Bogenschutz, M. P., Forcehimes, A. A., Pommy, J. A., Wilcox, C. E., Barbosa, P. C. R., \& Strassman, R. J. (2015). Psilocybin-assisted treatment for alcohol dependence: a proof-ofconcept study. Journal of psychopharmacology, 29(3), 289-299.

${ }^{23}$ Ross, S. (2012). Serotonergic hallucinogens and emerging targets for addiction pharmacotherapies. Psychiatric Clinics, 35(2), 357-374. 
486

487

488

489

490

491

492

493

494

495

496

497

498

499

500

501

502

503

504

505

506

507

508

509

510

511

512

513

514

515

516

${ }^{24}$ Johnson, M.W., Garcia-Romeu, A., Griffiths, R.R. (2016). Long-term follow-up of psilocybin-facilitated smoking cessation. Am. J. Drug Alcohol Abuse 43, 55-60. https:// doi.org/10.3109/00952990.2016.1170135. Johnson, M.W., Garcia-Romeu, A., Griffiths, R.R. (2016). Long-term follow-up of psilocybin-facilitated smoking cessation. Am. J. Drug Alcohol Abuse 43, 55-60. https:// doi.org/10.3109/00952990.2016.1170135.

${ }^{25}$ Barrett, F. S., Doss, M. K., Sepeda, N. D., Pekar, J. J., \& Griffiths, R. R. (2020). Emotions and brain function are altered up to one month after a single high dose of psilocybin. Scientific reports, $10(1), 1-14$.

${ }^{26}$ Griffiths, R. R., Johnson, M. W., Richards, W. A., Richards, B. D., Jesse, R., MacLean, K. A., ... \& Klinedinst, M. A. (2018). Psilocybin-occasioned mystical-type experience in combination with meditation and other spiritual practices produces enduring positive changes in psychological functioning and in trait measures of prosocial attitudes and behaviors. Journal of Psychopharmacology, 32(1), 49-69.

${ }^{27}$ Nicholas, C. R., Henriquez, K. M., Gassman, M. C., Cooper, K. M., Muller, D., Hetzel, S., ... \& Hutson, P. R. (2018). High dose psilocybin is associated with positive subjective effects in healthy volunteers. Journal of psychopharmacology, 32(7), 770-778.

${ }^{28}$ Lyons, T., \& Carhart-Harris, R. L. (2018). Increased nature relatedness and decreased authoritarian political views after psilocybin for treatment-resistant depression. Journal of Psychopharmacology, 32(7), 811-819.

${ }^{29}$ Griffiths, R. R., Richards, W. A., Johnson, M. W., McCann, U. D., \& Jesse, R. (2008). Mystical-type experiences occasioned by psilocybin mediate the attribution of personal meaning and spiritual significance 14 months later. Journal of psychopharmacology, 22(6), 621-632. 
$517{ }^{30}$ Griffiths, R. R., Johnson, M. W., Richards, W. A., Richards, B. D., McCann, U., \& Jesse, R.

518 (2011). Psilocybin occasioned mystical-type experiences: immediate and persisting dose-

519 related effects. Psychopharmacology, 218(4), 649-665.

520

$521{ }^{31}$ Schmid, Y., \& Liechti, M. E. (2018). Long-lasting subjective effects of LSD in normal

522 subjects. Psychopharmacology, 235(2), 535-545.

523

524

${ }^{32}$ Griffiths, R. R., Richards, W. A., McCann, U., \& Jesse, R. (2006). Psilocybin can occasion

525 mystical-type experiences having substantial and sustained personal meaning and spiritual significance. Psychopharmacology, 187(3), 268-283.

527

528

529

${ }^{33}$ Watts, R., Day, C., Krzanowski, J., Nutt, D., \& Carhart-Harris, R. (2017). Patients' accounts of increased "connectedness" and "acceptance" after psilocybin for treatment-resistant depression. Journal of humanistic psychology, 57(5), 520-564.

532

533

${ }^{34}$ Lebedev, A. V., Kaelen, M., Lövdén, M., Nilsson, J., Feilding, A., Nutt, D. J., \& CarhartHarris, R. L. (2016). LSD-induced entropic brain activity predicts subsequent personality change. Human brain mapping, 37(9), 3203-3213.

536

${ }^{35}$ MacLean, K. A., Johnson, M. W., \& Griffiths, R. R. (2011). Mystical experiences occasioned 538 by the hallucinogen psilocybin lead to increases in the personality domain of openness. Journal of Psychopharmacology, 25(11), 1453-1461.

${ }^{36}$ Netzband, N., Ruffell, S., Linton, S., Tsang, W. F., \& Wolff, T. (2020). Modulatory effects of ayahuasca on personality structure in a traditional framework. Psychopharmacology, 237(10), 3161-3171.

${ }^{37}$ Terracciano, A., McCrae, R. R., Brant, L. J., \& Costa Jr, P. T. (2005). Hierarchical linear modeling analyses of the NEO-PI-R scales in the Baltimore Longitudinal Study of Aging. Psychology and aging, 20(3), 493. 
$549{ }^{38}$ Pokorny, T., Preller, K. H., Kometer, M., Dziobek, I., \& Vollenweider, F. X. (2017). Effect of

550 psilocybin on empathy and moral decision-making. International Journal of

551 Neuropsychopharmacology, 20(9), 747-757.

552

$553{ }^{39}$ Lyons, T., \& Carhart-Harris, R. L. (2018). More realistic forecasting of future life events

554 after psilocybin for treatment-resistant depression. Frontiers in psychology, 9, 1721.

555

556

${ }^{40}$ Noorani, T., Garcia-Romeu, A., Swift, T. C., Griffiths, R. R., \& Johnson, M. W. (2018).

557 Psychedelic therapy for smoking cessation: qualitative analysis of participant

558 accounts. Journal of Psychopharmacology, 32(7), 756-769.

559

560

${ }^{41}$ Corlett, P. R., Taylor, A. H., Wang, X.-J., Fletcher, P. C., and Krystal, J. H. (2010). Toward a 561 neurobiology of delusions. Progress in Neurobiology, 92, 345-369.

562

563

${ }^{42}$ Connors, M. H., \& Halligan, P. W. (2015). A cognitive account of belief: a tentative road 564 map. Frontiers in psychology, 5, 1588.

565

566

${ }^{43}$ Schwitzgebel, E. (2006). Belief. URL =

567

<https://plato.stanford.edu/archives/fall2019/entries/belief/>.

568

569

${ }^{44}$ Buchanan, R. (2012). Is belief a propositional attitude? Philosophers Imprint, 12(1), 1-20.

570

571

${ }^{45}$ Williams, D. (2020). Socially adaptive belief. Mind \& Language. doi: 10.1111/mila.12294

572

$573{ }^{46}$ Grzankowski, A., \& Buchanan, R. (2019). Propositions on the Cheap. Philosophical Studies, 574 176(12), 3159-3178.

575

576

${ }^{47}$ White, A. S., Hacquard, V., \& Lidz, J. (2018). Semantic information and the syntax of

577 propositional attitude verbs. Cognitive science, 42(2), 416-456.

578

$579{ }^{48}$ de Villiers, J. (2020). With Language in Mind. Language Learning and Development, 1-25. 580 
581

582

583

584

585

586

587

588

589

590

591

592

593

594

595

596

597

598

599

600

601

602

603

604

605

606

607

608

609

610

611

612

613

${ }^{49}$ Burge, T. (2018). Do infants and nonhuman animals attribute mental states? Psychological Review, 125(3), 409.

${ }^{50}$ Newen, A., \& Starzak, T. (2020). How to ascribe beliefs to animals. Mind \& Language.

${ }^{51}$ Aguilera, M. (2018). Why the content of animal thought cannot be propositional. Analisis Filosofico, 38(2), 183-207.

52 Boisseau, R. P., Vogel, D., \& Dussutour, A. (2016). Habituation in non-neural organisms: evidence from slime moulds. Proceedings of the Royal Society B: Biological Sciences, 283(1829), 20160446.

${ }^{53}$ Smith-Ferguson, J., \& Beekman, M. (2020). Who needs a brain? Slime moulds, behavioural ecology and minimal cognition. Adaptive Behavior, 28(6), 465-478.

${ }^{54}$ Gawronski, B., Bodenhausen, G. V., Mitchell, C. J., De Houwer, J., \& Lovibond, P. F. (2009). Operating principles versus operating conditions in the distinction between associative and propositional processes. Behavioral and Brain Sciences, 32(2), 207.

${ }^{55}$ De Houwer, J. (2009). The propositional approach to associative learning as an alternative for association formation models. Learning \& Behavior, 37(1), 1-20.

${ }^{56}$ Klein-Flügge, M. C., Wittmann, M. K., Shpektor, A., Jensen, D. E., \& Rushworth, M. F. (2019). Multiple associative structures created by reinforcement and incidental statistical learning mechanisms. Nature communications, 10(1), 1-15.

${ }^{57}$ Spicer, S. G., Mitchell, C. J., Wills, A. J., \& Jones, P. M. (2020). Theory protection in associative learning: Humans maintain certain beliefs in a manner that violates prediction error. Journal of Experimental Psychology: Animal Learning and Cognition, 46(2), 151.

${ }^{58}$ Corlett, P. R., Aitken, M. R., Dickinson, A., Shanks, D. R., Honey, G. D., Honey, R. A., Robbins, T.W., Bullmore, E.T., \& Fletcher, P. C. (2004). Prediction error during retrospective 
614 revaluation of causal associations in humans: $\mathrm{fMRI}$ evidence in favor of an associative model

615 of learning. Neuron, 44(5), 877-888.

616

61759 Terao, K., Matsumoto, Y., \& Mizunami, M. (2015). Critical evidence for the prediction

618 error theory in associative learning. Scientific reports, 5(1), 1-8.

619

$620{ }^{60}$ Schultz, W., Tremblay, L., \& Hollerman, J. R. (1998). Reward prediction in primate basal

621 ganglia and frontal cortex. Neuropharmacology, 37(4-5), 421-429.

622

$623{ }^{61}$ Schultz, W., Tremblay, L., \& Hollerman, J. R. (2000). Reward processing in primate

624 orbitofrontal cortex and basal ganglia. Cerebral cortex, 10(3), 272-283.

625

$626{ }^{62}$ Alikaya, A., Rack-Wildner, M., \& Stauffer, W. R. (2018). Reward and value coding by

627 dopamine neurons in non-human primates. Journal of Neural Transmission, 125(3), 565-

628574.

629

630

${ }^{63}$ Tanaka, S., O'Doherty, J. P., \& Sakagami, M. (2019). The cost of obtaining rewards

631 enhances the reward prediction error signal of midbrain dopamine neurons. Nature

632 communications, 10(1), 1-13.

633

634

${ }^{64}$ Laurent, V., \& Balleine, B. W. (2015). Factual and counterfactual action-outcome

635 mappings control choice between goal-directed actions in rats. Current Biology, 25(8), 1074-

636 1079.

637

$638{ }^{65}$ Wise, R. A. (2004). Dopamine, learning and motivation. Nature reviews neuroscience, 5(6), 639 483-494.

640

$641{ }^{66}$ Puig, M., Rose, J., Schmidt, R., \& Freund, N. (2014). Dopamine modulation of learning and

642 memory in the prefrontal cortex: insights from studies in primates, rodents, and birds.

643 Frontiers in neural circuits, 8, 93. 
$645{ }^{67}$ Tomov, M. S., Dorfman, H. M., \& Gershman, S. J. (2018). Neural computations underlying 646 causal structure learning. Journal of Neuroscience, 38(32), 7143-7157.

647

$648{ }^{68}$ Nour, M. M., Dahoun, T., Schwartenbeck, P., Adams, R. A., FitzGerald, T. H., Coello, C., Ball, 649 M.B., Dolan, R.J., \& Howes, O. D. (2018). Dopaminergic basis for signaling belief updates, but 650 not surprise, and the link to paranoia. Proceedings of the National Academy of 651 Sciences, 115(43), E10167-E10176.

$652{ }^{69}$ Nasser, H. M., Calu, D. J., Schoenbaum, G., \& Sharpe, M. J. (2017). The dopamine 653 prediction error: contributions to associative models of reward learning. Frontiers in 654 psychology, 8, 244.

655 656 ${ }^{70}$ Friston, K. (2003). Learning and inference in the brain. Neural Networks, 16(9), 1325-1352.

657

658

${ }^{71}$ Friston, K., FitzGerald, T., Rigoli, F., Schwartenbeck, P., \& Pezzulo, G. (2016). Active 659 inference and learning. Neuroscience \& Biobehavioral Reviews, 68, 862-879.

660

661

${ }^{72}$ Corlett, P. R., Honey, G. D., \& Fletcher, P. C. (2016). Prediction error, ketamine and 662 psychosis: An updated model. Journal of psychopharmacology, 30(11), 1145-1155.

663

664

${ }^{73}$ Corlett, P. R., Frith, C. D., \& Fletcher, P. C. (2009). From drugs to deprivation: a Bayesian 665 framework for understanding models of psychosis. Psychopharmacology, 206(4), 515-530. 666 667 ${ }^{74}$ Carhart-Harris, R. L., \& Nutt, D. J. (2017). Serotonin and brain function: a tale of two 668 receptors. Journal of Psychopharmacology, 31(9), 1091-1120.

669

670

${ }^{75}$ Swanson, L. R. (2018). Unifying theories of psychedelic drug effects. Frontiers in 671 pharmacology, 9, 172.

672

674 "REBUS and the Anarchic Brain: Toward a Unified Model of the Brain Action of 675 Psychedelics".

676 
$677{ }^{77}$ Friston K. (2002). Functional integration and inference in the brain. Progress in 678 neurobiology, 68(2), 113-143.

679

680

681

682

${ }^{78}$ Von Helmholtz $\mathrm{H}$. The facts of perception. In: Kahl R (ed.). Selected Writings of Herman von Helmholtz. Weslyan University Press, 1878.

683

$684{ }^{79}$ Kersten D, Mamassian P, Yuille A. Object perception as Bayesian inference. Annu Rev 685 Psychol 2004;55:271-304.

686

687

${ }^{80}$ Körding KP, Wolpert DM. Bayesian integration in sensorimotor learning. Nature $688 \quad 2004 ; 427: 244-7$.

689

690

${ }^{81}$ Friston KJ, Stephan KE. Free-energy and the brain. Synthese 2007;159:417-58.

691

692

${ }^{82}$ Hohwy, J. (2013). The predictive mind. Oxford University Press.

693

694

${ }^{83}$ Clark, A. (2013). Whatever next? Predictive brains, situated agents, and the future of 695 cognitive science. Behavioral and brain sciences, 36(3), 181-204.

696

697

${ }^{84}$ Powers, A. R., III, Kelley, M., \& Corlett, P. R. (2016). Hallucinations as top-down effects on perception. Biological psychiatry. Cognitive neuroscience and neuroimaging, 1(5), 393-400.

699

700

${ }^{85}$ Sterzer P, Adams RA, Fletcher P et al. The Predictive Coding Account of Psychosis.

701

Biological Psychiatry 2018:1-10.

702

703

${ }^{86}$ Corlett, P. R., Horga, G., Fletcher, P. C., Alderson-Day, B., Schmack, K., \& Powers, A. R., 3rd

704

(2019). Hallucinations and Strong Priors. Trends in cognitive sciences, 23(2), 114-127.

705

706

${ }^{87}$ Clifford, C. W., Webster, M. A., Stanley, G. B., Stocker, A. A., Kohn, A., Sharpee, T. O., \&

707 Schwartz, O. (2007). Visual adaptation: neural, psychological and computational aspects.

Vision research, 47(25), 3125-3131. 
${ }^{88}$ Kiebel, S. J., Daunizeau, J., \& Friston, K. J. (2008). A hierarchy of time-scales and the brain.

PLoS computational biology, 4(11), e1000209.

712

${ }^{89}$ Summerfield, C., \& de Lange, F. P. (2014). Expectation in perceptual decision making:

${ }^{90}$ Friston K. (2008). Hierarchical models in the brain. PLoS computational biology, 4(11), e1000211.

718

${ }^{91}$ Felleman, D. J., \& Van Essen, D. C. (1991). Distributed hierarchical processing in the primate cerebral cortex. Cerebral cortex (New York, N.Y. : 1991), 1(1), 1-47.

721

92 Bastos, A. M., Usrey, W. M., Adams, R. A., Mangun, G. R., Fries, P., \& Friston, K. J. (2012). Canonical microcircuits for predictive coding. Neuron, 76(4), 695-711.

724

${ }^{93}$ Vetter, P., \& Newen, A. (2014). Varieties of cognitive penetration in visual perception.

727

${ }^{94}$ Macpherson F. (2017). The relationship between cognitive penetration and predictive coding. Consciousness and cognition, 47, 6-16.

730

${ }^{95}$ O'Callaghan, C., Kveraga, K., Shine, J. M., Adams, R. B., Jr, \& Bar, M. (2017). Predictions penetrate perception: Converging insights from brain, behaviour and disorder. Consciousness and cognition, 47, 63-74.

${ }^{96}$ Gold, J. I., \& Stocker, A. A. (2017). Visual Decision-Making in an Uncertain and Dynamic World. Annual review of vision science, 3, 227-250. 
${ }^{98}$ Fischer, J., \& Whitney, D. (2014). Serial dependence in visual perception. Nature neuroscience, 17(5), 738-743.

${ }^{99}$ Liberman, A., Fischer, J., \& Whitney, D. (2014). Serial dependence in the perception of faces. Current biology : CB, 24(21), 2569-2574.

747

${ }^{100}$ St John-Saaltink, E., Kok, P., Lau, H. C., \& de Lange, F. P. (2016). Serial Dependence in

Perceptual Decisions Is Reflected in Activity Patterns in Primary Visual Cortex. The Journal of neuroscience : the official journal of the Society for Neuroscience, 36(23), 6186-6192.

751

${ }^{101}$ Fritsche, M., Mostert, P., \& de Lange, F. P. (2017). Opposite Effects of Recent History on 753 Perception and Decision. Current biology : CB, 27(4), 590-595.

754

755

102 Kiyonaga, A., Scimeca, J. M., Bliss, D. P., \& Whitney, D. (2017). Serial Dependence across 756 Perception, Attention, and Memory. Trends in cognitive sciences, 21(7), 493-497.

757

758

103 Papadimitriou, C., White, R. L., 3rd, \& Snyder, L. H. (2017). Ghosts in the Machine II:

759 Neural Correlates of Memory Interference from the Previous Trial. Cerebral cortex (New 760 York, N.Y. : 1991), 27(4), 2513-2527.

761

762

${ }^{104}$ Carhart-Harris, R. L., Roseman, L., Haijen, E., Erritzoe, D., Watts, R., Branchi, I., \& Kaelen, 763 M. (2018). Psychedelics and the essential importance of context. Journal of psychopharmacology (Oxford, England), 32(7), 725-731.

765 and contextual factors in the use of psychedelics. Transcultural Psychiatry. beverage Ayahuasca on binocular rivalry: interhemispheric switching or interhemispheric fusion?. Journal of psychoactive drugs, 35(3), 367-374. 
$773{ }^{107}$ Frecska, E., White, K. D., \& Luna, L. E. (2004). Effects of ayahuasca on binocular rivalry 774 with dichoptic stimulus alternation. Psychopharmacology, 173(1-2), 79-87.

775

776

${ }^{108}$ Carter, O. L., Pettigrew, J. D., Hasler, F., Wallis, G. M., Liu, G. B., Hell, D., \& Vollenweider,

777 F. X. (2005). Modulating the rate and rhythmicity of perceptual rivalry alternations with the mixed 5-HT2A and 5-HT1A agonist psilocybin. Neuropsychopharmacology : official

779 publication of the American College of Neuropsychopharmacology, 30(6), 1154-1162

780

781

${ }^{109}$ Carter, O. L., Hasler, F., Pettigrew, J. D., Wallis, G. M., Liu, G. B., \& Vollenweider, F. X.

782 (2007). Psilocybin links binocular rivalry switch rate to attention and subjective arousal levels in humans. Psychopharmacology, 195(3), 415-424.

784

785

${ }^{110}$ Leptourgos, P., Bouttier, V., Jardri, R., \& Denève, S. (2020). A functional theory of bistable 786 perception based on dynamical circular inference. PLoS computational biology, 16(12), e1008480.

788

789

${ }^{111}$ Heyes, C. (2016). Who knows? Metacognitive social learning strategies. Trends in cognitive sciences, 20(3), 204-213.

791

792

${ }^{112}$ Benjamin Disraeli, 1804-1881, British Prime Minister (Tory), Lothair

793

Eigen's Political and Historical Quotation

794

795

${ }^{113}$ Lovibond, P. F. (2004). Cognitive processes in extinction. Learning \& Memory, 11(5), 495796 500.

797

798

${ }^{114}$ Duerler, P., Schilbach, L., Stämpfli, P., Vollenweider, F. X., \& Preller, K. H. (2020). LSD-

799 induced increases in social adaptation to opinions similar to one's own are associated with stimulation of serotonin receptors. Scientific reports, 10(1), 1-11.

801

${ }^{115}$ Carhart-Harris, R. L., Kaelen, M., Whalley, M. G., Bolstridge, M., Feilding, A., \& Nutt, D. J.

803 (2015). LSD enhances suggestibility in healthy volunteers. Psychopharmacology, 232(4), 785804794. 
805

806

${ }^{116}$ Patterson DR, Hoffer C, Jensen MP, Wiechman SA, Sharar SR. (2010). Ketamine as a

807 possible moderator of hypnotizability: A feasibility study. International Journal of Clinical Experimental Hypnosis, 66(3, 298-307.

809

${ }^{117}$ Olson, J. A., Suissa-Rocheleau, L., Lifshitz, M., Raz, A., \& Veissière, S. P. (2020). Tripping on nothing: placebo psychedelics and contextual factors. Psychopharmacology, 237(5), 13711382.

813

${ }^{118}$ Dos Santos, R. G., \& Hallak, J. E. C. (2020). Therapeutic use of serotoninergic hallucinogens: a review of the evidence and of the biological and psychological mechanisms. Neuroscience \& Biobehavioral Reviews, 108, 423-434.

817

119 Erritzoe, D., Roseman, L., Nour, M. M., MacLean, K., Kaelen, M., Nutt, D. J., \& CarhartHarris, R. L. (2018). Effects of psilocybin therapy on personality structure. Acta Psychiatrica Scandinavica, 138(5), 368-378.

821

${ }^{120}$ Griffiths, R. R., Johnson, M. W., Richards, W. A., Richards, B. D., Jesse, R., MacLean, K. A., ... \& Klinedinst, M. A. (2018). Psilocybin-occasioned mystical-type experience in combination with meditation and other spiritual practices produces enduring positive changes in psychological functioning and in trait measures of prosocial attitudes and behaviors. Journal of Psychopharmacology, 32(1), 49-69.

${ }^{121}$ Hood, R. W., Jr., Ghorbani, N., Watson, P. J., Ghramaleki, A. F., Bing, M. N., Davison, H. K., Morris, R. J., \& Williamson, W. P. (2001). Dimensions of the Mysticism Scale: Confirming the three-factor structure in the United States and Iran. Journal for the Scientific Study of Religion, 40(4), 691-705. mystical-type experiences having substantial and sustained personal meaning and spiritual significance. Psychopharmacology, 187(3), 268-283. 
837

838

839

840

841

842

843

844

845

846

847

848

849

850

851

852

853

854

855

856

857

858

859

860

861

862

863

864

865

866

867

868

${ }^{123}$ Strassman, R. J., Qualls, C. R., Uhlenhuth, E. H., \& Kellner, R. (1994). Dose-response study of $\mathrm{N}, \mathrm{N}$-dimethyltryptamine in humans: II. Subjective effects and preliminary results of a new rating scale. Archives of general psychiatry, 51(2), 98-108.

${ }^{124}$ Griffiths, R. R., Johnson, M. W., Richards, W. A., Richards, B. D., McCann, U., \& Jesse, R. (2011). Psilocybin occasioned mystical-type experiences: immediate and persisting doserelated effects. Psychopharmacology, 218(4), 649-665.

${ }^{125}$ Li, D., \& Mashour, G. A. (2019). Cortical dynamics during psychedelic and anesthetized states induced by ketamine. Neuroimage, 196, 32-40.

${ }^{126}$ Kohtala, S., \& Rantamäki, T. (2021). Rapid-acting antidepressants and the regulation of TrkB neurotrophic signalling-insights from ketamine, nitrous oxide, seizures, and anaesthesia. Basic \& Clinical Pharmacology \& Toxicology.

${ }^{127}$ Halberstadt, A. L., \& Geyer, M. A. (2011). Multiple receptors contribute to the behavioral effects of indoleamine hallucinogens. Neuropharmacology, 61(3), 364-381.

${ }^{128}$ Madsen, M. K., Fisher, P. M., Burmester, D., Dyssegaard, A., Stenbæk, D. S., Kristiansen, S., Johansen, S. S., Lehel, S., Linnet, K., Svarer, C., Erritzoe, D., Ozenne, B., \& Knudsen, G. M. (2019). Psychedelic effects of psilocybin correlate with serotonin 2A receptor occupancy and plasma psilocin levels. Neuropsychopharmacology : official publication of the American College of Neuropsychopharmacology, 44(7), 1328-1334.

${ }^{129}$ Skryabin, V. Y., Vinnikova, M., Nenastieva, A., \& Alekseyuk, V. (2018). Hallucinogen persisting perception disorder: a literature review and three case reports. Journal of addictive diseases, 37(3-4), 268-278.

${ }^{130}$ Martinotti, G., Santacroce, R., Pettorruso, M., Montemitro, C., Spano, M. C., Lorusso, M., ... \& Lerner, A. G. (2018). Hallucinogen persisting perception disorder: etiology, clinical features, and therapeutic perspectives. Brain sciences, 8(3), 47. 
${ }^{131}$ Powers, A. R., Mathys, C., \& Corlett, P. R. (2017). Pavlovian conditioning-induced

871 hallucinations result from overweighting of perceptual priors. Science, 357(6351), 596-600.

872

873

${ }^{132}$ Colpaert, F. C., \& Janssen, P. A. J. (1983). A characterization of LSD-antagonist effects of pirenperone in the rat. Neuropharmacology, 22(8), 1001-1005.

876

${ }^{133}$ Colpaert, F. C., Niemegeers, C. J., \& Janssen, P. A. (1982). A drug discrimination analysis of lysergic acid diethylamide (LSD): in vivo agonist and antagonist effects of purported 5hydroxytryptamine antagonists and of pirenperone, a LSD-antagonist. Journal of Pharmacology and Experimental Therapeutics, 221(1), 206-214.

881

${ }^{134}$ Sershen, H., Hashim, A., \& Lajtha, A. (2000). Serotonin-mediated striatal dopamine release involves the dopamine uptake site and the serotonin receptor. Brain research bulletin, 53(3), 353-357.

885

${ }^{135}$ Nowak, P., Szczerbak, G., Biedka, I., Drosik, M., Kostrzewa, R. M., \& Brus, R. (2006). Effect of Ketanserin and Amphetamine on Nigrostriatial Transmission and Reactive Oxygen Species in Parkinsonian Rats in Vivo Microdialysis Study. Journal of physiology and pharmacology, 57(4), 583-597.

890

${ }^{136}$ Morici, J. F., Bekinschtein, P., \& Weisstaub, N. V. (2015). Medial prefrontal cortex role in recognition memory in rodents. Behavioural Brain Research, 292, 241-251. preferential involvement of 5-HT2A serotonin receptors in stress-and drug-induced dopamine release in the rat medial prefrontal cortex. Neuropsychopharmacology, 31(2), 265-277. 
901

902

$903{ }^{139}$ Klee G., Weintraub W. Neuro-Psychopharmacology. Elsevier-Van Nostrand; Princeton, NJ, 904 USA: 1959. Paranoid response following lysergic acid diethylamide (LSD-25) pp. 457-460.

905

$906{ }^{140}$ Goff, P., Seager, W., \& Allen-Hermanson, S. (2001). Panpsychism.

907

$908{ }^{141}$ Metraux, D. A. (1995). Religious terrorism in Japan: The fatal appeal of Aum Shinrikyo.

909 Asian Survey, 35(12), 1140-1154.

910

911 